Article

\title{
Numerical and Physical Simulation of Heat Transfer Enhancement Using Oval Dimple Vortex Generators-Review and Recommendations
}

\author{
Alexander Mironov ${ }^{1}$, Sergey Isaev ${ }^{2}$, Artem Skrypnik ${ }^{1}$ and Igor Popov ${ }^{1, * \mathbb{D}}$ \\ 1 Department of Heat Engineering and Power Machinery, Kazan National Research Technical University \\ named after A. N. Tupolev-KAI, 10 K.Marx.str., 420111 Kazan, Russia; alexcander1993@mail.ru (A.M.); \\ anskrypnik@kai.ru (A.S.) \\ 2 Department of Aero and Aircraft Flight Dynamics, Saint Petersburg State University of Civil Aviation, \\ 38 Pilotov Street, 196210 Saint Petersburg, Russia; isaev3612@yandex.ru \\ * Correspondence: popov-igor-alex@yandex.ru; Tel.: +7-919-644-1609
}

Received: 10 August 2020; Accepted: 25 September 2020; Published: 9 October 2020

\begin{abstract}
Vortex generation and flow disruption in heat exchanger passages by means of surface modification is a widely used passive heat transfer augmentation technique. The present paper contains the results of numerical and experimental studies of the hydraulic resistance and heat transfer in the rectangle duct with oval-trench- and oval-arc-shaped dimples applied to the heat transfer surface. For the turbulent flow in the duct $\left(\operatorname{Pr}=0.71, \operatorname{Re}_{d}=3200-9 \times 10^{4}\right.$-for heat transfer determination and $R e_{d}=500-10^{4}$-for the friction factor measurements), rational geometrical parameters of the oval-trench dimple were determined: relative elongation of dimple $l / b=5.57-6.78$ and relative depth $l / b=5.57-6.78$, while the value of the attack angle to the mean flow was fixed $\varphi=(45-60)^{\circ}$. The comparison of the experimental and numerical modeling for the flow in the narrow duct over the surface with a single- and multi-row dimple arrangement has revealed a good agreement. It was found that the average heat transfer coefficient magnitudes in such ducts could be increased 1.5-2.5 times by means of single and multi-row dimple application on the heat transfer surface. The heat transfer augmentation for the surfaces with the oval-arched dimples was found to be $10 \%$ greater than the one for the oval-trench dimples. The corresponding friction factor augmentation was found to be $125-300 \%$ in comparison to the smooth surface duct. The obtained experimental data were used for the data generalization. Derived generalized equation allows for predicting the friction factor and heat transfer coefficient values for the flow over the single-row oval-trench simple arrangement. The maximal deviation of the experimental data from the proposed equations was found to be $20 \%$. The application of the artificial neural networks for predicting the hydraulic resistance and heat transfer augmentation in such ducts was presented.
\end{abstract}

Keywords: heat transfer enhancement; hydraulic resistance; efficiency; numerical simulation; experiment; vortex generators; oval dimples

\section{Introduction}

Application of symmetric and asymmetric spherical shaped dimples on heat transfer surfaces is a method for heat transfer augmentation extensively investigated over the last decades [1-8]. Intensive vortex generation in the flow over such dimpled surfaces accompanied by the intensive generation of turbulence in the boundary layer and flow detachment significantly affects the aerodynamic characteristics of the flow as well as the heat and mass transfer near the wall.

At present, the primary objective is to determine the optimal dimple shape and its row arrangements on the surface: dimple width, depth, a dimple notching step, and attack angle values. 
Such optimal dimple geometrical parameters have to ensure heat transfer augmentation with the relatively low friction factor growth, in comparison to the flow over the smooth surface. Resolving this challenging issue by the researchers will be decisive for heat-exchange equipment production for manufacturers and customers. A fairly full overview of the experimental investigations on fluid flow and heat transfer in the ducts with dimpled surfaces for various technical applications is presented in [1]. The comprehensive review on the topic is presented in monographs $[9,10]$.

The use of numerical methods and specialized software has been shown a great success in modeling flow and heat transfer in various technical devices. Numerical simulations have sharply increased the research effectiveness, as it has reduced the costs associated with the establishment of thermophysical field experiments. It allows for estimating in detail the flow characteristics at various conditions (flow regimes, boundary conditions, thermal and physical properties of coolants, etc.) and different geometrical parameters (size, shape, and arrangement of dimples, etc.). However, numerical simulations findings have to be proved and verified with the results obtained experimentally. In addition, it is necessary to determine the rational dimensions of vortex generators for maximum heat transfer enhancement and maximum increase in thermal hydraulic efficiency, to propose engineering methods for determining heat transfer coefficients and hydraulic resistance coefficients for these ranges of parameters of vortex generators, and to investigate the possibility of using a neural network model to improve the accuracy of determining heat transfer coefficients and hydraulic resistance coefficients.

\section{Review of Numerical Simulation of Heat Transfer Enhancement Using Elongated Oval Dimples}

The oval-trench dimple consists of the cylindrical part with the length 1 and to halves of the spherical dimple with radius b placed at the cylindrical part ends (Figure 1). Thus, the width of the oval-trench dimple is equal to $b$. The dimple is applied to the heat transfer surface by the embedding of a sphere with the radius $R$ (Figure 1) on the depth $h$.
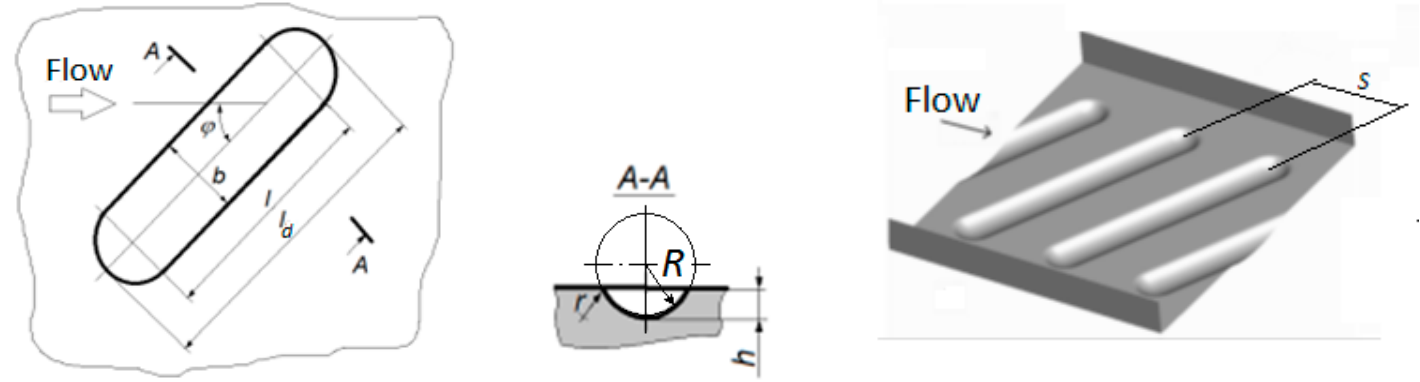

Figure 1. Characteristic scheme of the oval-trench-shaped dimple: $l$-a length of the dimple trench part, $\mathrm{m} ; l_{d}$ - a total length, $\mathrm{m} ; h$-a depth, $\mathrm{m} ; b$ - a width, $\mathrm{m} ; r$ - edge rounding radius, $\mathrm{m} ; \varphi$ —-the angle of attack of the dimple (to the main flow), deg; $S$-a notching step; $R$-an embedding sphere radius, $\mathrm{m}$; $A-A$-cross-section.

Varying the sphere embedding the value of the relative dimple depth $h / b$ could be changed. The relative dimple depth varies within the 0 to 0.5 and reaches its maximum for $h=R$ (and, therefore, $b=2 R$ ). Depending on the groove application technique, the conjugation of the groove and the flat surface the value of the rounding radius $\mathrm{r}$ varies. During the machining, the magnitude of $r$ could be kept $r=0$, but, during the molding or cold pressing, the value of $r$ increases. In the numerical simulation, the relative rounding was kept constant $r / b=0.21$.

The primary results on the flow over the elongated oval dimples are presented in [11-16].

The Reynolds number in those works was based on the value of mean flow velocity, $\bar{V}=m /(\rho \cdot A)$ and the duct height: hch, $R e=\rho \bar{V} h_{c h} / \mu$, where $\rho$-the density of the fluid, where $A$-area of the cross-section of the duct, and $\mu$-fluid dynamic viscosity. It should be noticed that the value of the Nusselt number $\mathrm{Nu}$ for the smooth duct (subindex 0 ) and the duct with the dimpled surface was 
calculated on the base of the duct height: $N u=\alpha h_{c h} / \lambda$ and $N u=\alpha_{0} h_{c h} / \lambda$, where $\alpha$ and $\alpha_{0}$ heat transfer coefficients, $\lambda$ fluid heat conductivity. The heat transfer coefficient was calculated for a characteristic surface section with the dimple of the length $L=7 h_{c h}$ and $B=9 h_{c h}$ and periodic boundary conditions. Thus, the heat transfer coefficients on the dimpled surface and on the surrounding flat surface were considered. The smooth heat transfer coefficient $\alpha_{0}$ was calculated on the surface of the same area. The temperature of the surface was kept constant $T=303 \mathrm{~K}$. Darcy-Weisbach friction factor values were defined analogously on the basis of the value of a pressure loss $\Delta P-\xi=2 \cdot \Delta P \cdot h_{c h} /(\rho \cdot \bar{V} L)$.

Isaev S.A. et al. in $[12,13]$ have presented numerical results of turbulent water flow over the oval-trench dimple inclined to the main flow by the angle of $\varphi=45^{\circ}$. The value of the Reynolds number Re in the simulations remained constant $R e=10^{4}$.

The value of the spot area of the dimple and the relative depth of the dimple $h / b$ was kept constant while the ratio of the length of the dimple to the simple width $l_{d} / b$ was varied. In the referenced works was shown that the hydraulic loss on the test domain is rising with the increase in the $l_{d} / b$ value and reaches the maximum at the $l_{d} / b=3$. The further increase in the value of the $l_{d} / b$ ratio leads to the monotonically decreasing of the hydraulic losses up to the value of the reference test section with the baseline spherical dimple (Figure 2). The heat transfer augmentation $N u / N u_{0}$ is monotonically increasing with the growth of the $l_{d} / b$ value. This fact is linked to the secondary flow structure in the dimple. The $l_{d} / b$ value growth for the elongated oval dimple, inclined to the main flow, the secondary flow in the dimple accelerates, wherein the maximum value of the transverse velocity referred to the average mass flow velocity in the channel, reaches the value of 0.85 at $l_{d} b=6-7$.

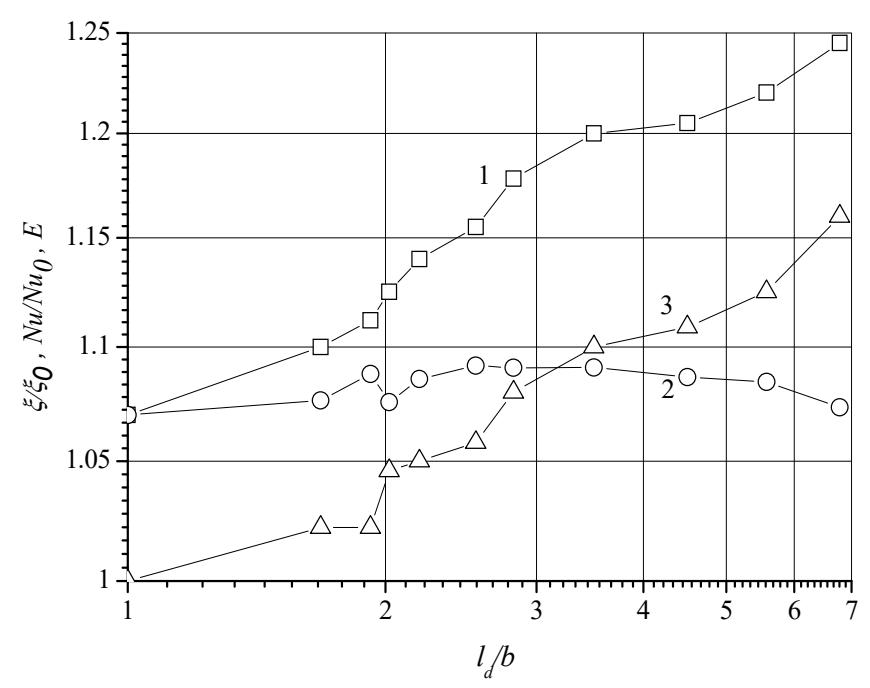

Figure 2. Relative heat transfer $N u / N u_{0}$ (designation 1), friction factor $\xi / \xi_{0}$ (designation 2) ratio, and thermal-hydraulic efficiency $E=\left(N u / N u_{0}\right) /\left(\xi / \xi_{0}\right)$ (3) along the length of the domain with the oval-trench dimple.

In the study of the turbulent water flow over the oval-trench dimple oriented at the attack angle $\varphi$ $=30-60^{\circ}$ to the main flow [14] has shown that the best thermal properties were found for the value of the relative elongation of the dimple $l_{d} / b=6$, and attack angle value of $\varphi=45^{\circ}$ (Figure 3). 


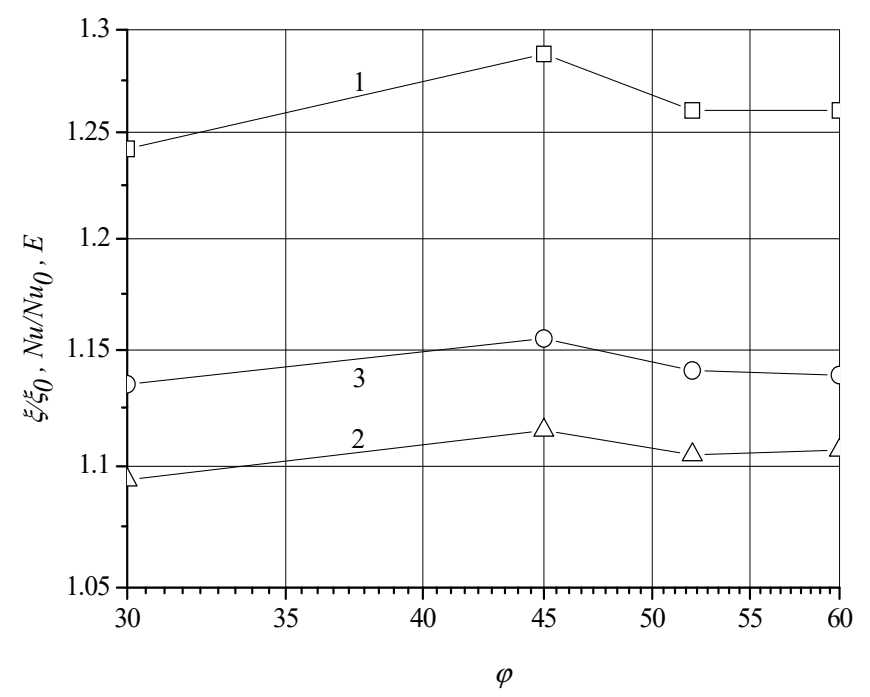

Figure 3. Relative heat transfer $N u / N u_{0}$ (1), friction factor $\xi / \xi_{0}(2)$ ratio, and thermal-hydraulic efficiency $E=\left(N u / N u_{0}\right) /\left(\xi / \xi_{0}\right)(3)$ versus the angle of attack $\varphi$ for the $l_{d} / b=6$.

The laminar airflow $\left(R e=10^{3}\right)$ in the narrow duct with one row of oval-trench dimples oriented at the attach angle of $\varphi=45^{\circ}$ to the main flow was studied in $[15,16]$.

By varying the relative depth of the dimple $h / b$ in the range $h / b=0.0625-0.375$, the 1.5-fold increase in the maximum flow speed for the $h / b$ value of $h / b=0.25$ (for $h / h_{c h}=0.25, h_{c h}$-height of the duct) was found.

It is shown that an increase in the relative depth of the depression from the value $h / b=0.0625$ to 0.375 leads to an increase from $N u / N u_{0}=1.1$ to 1.9 (Figure 4). The increase in the hydraulic resistance coefficient changes from $\xi / \xi_{0}=1$ to 1.42 . Therefore, the magnitude of thermohydraulic efficiency $E=\left(N u / N u_{0}\right) /\left(\xi / \xi_{0}\right)$, in the range $\mathrm{h} / \mathrm{b}$ from $h / b=0.0625$ to 0.25 , rises from the value of 1.1 to the 1.47. However, in the range of the relative depth $h / b=0.25-0.375$ the magnitude of $E$ decreases from 1.47 to 1.33. Thus, the observed optimal (maximum value of thermohydraulic efficiency) value of the relative depth of the oval-trench dimple is found to be $h / b=0.25$.

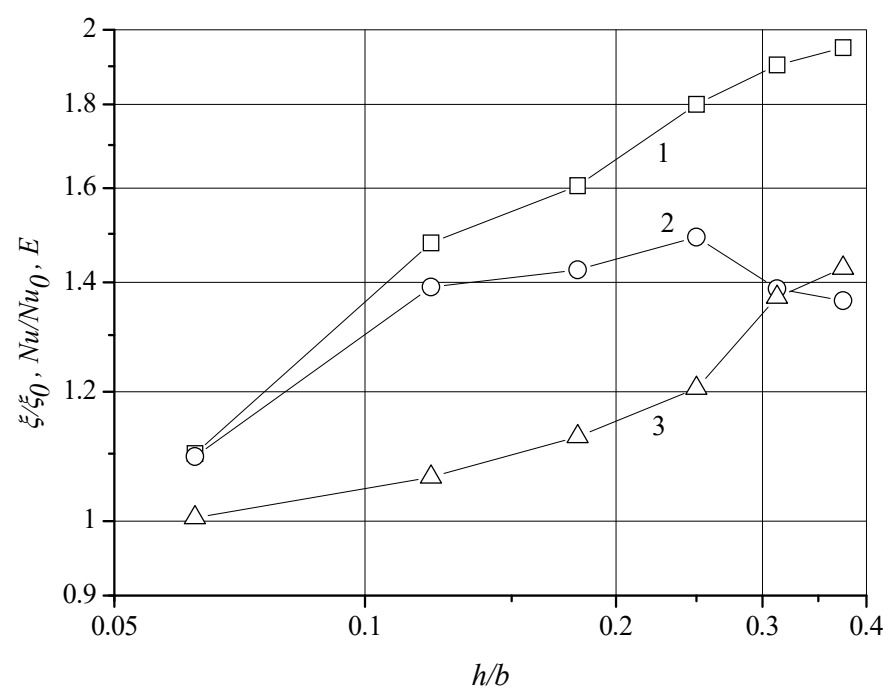

Figure 4. Relative heat transfer $N u / N u_{0}$ (1), friction factor $\xi / \xi_{0}$ (3) ratio, and thermal-hydraulic efficiency $E=\left(N u / N u_{0}\right) /\left(\xi / \xi_{0}\right)(2)$ versus the relative depth of the dimple $h / b$.

A comprehensive analysis of the results presented in [11-16] and in Figures 2-5 allows for determining the rational geometrical dimple parameters. The relative elongation of a such dimple shall 
vary in the range $l / b=5.57-6.78$ and relative depth of $h / b=0.18-0.37$, while the value of the attack angle to the mean flow is fixed $\varphi=45^{\circ}$ [17]. The oval-trench dimples with the proposed parameters promote the generation of mono-vortices in and after dimple. Those secondary flows enlarge the thermal performance of the whole heat transfer surface. Such a mono-vortex structure is highly sustainable and its intensity exceeds the value for the hemispherical dimple. I also noted the alternating of the secondary flow from the bimodal to the mono-vortex structure with the spike in the magnitude of the heat transfer rate. The major contribution of the dimple elongation on the heat transfer augmentation and the secondary flow reconstruction was observed.

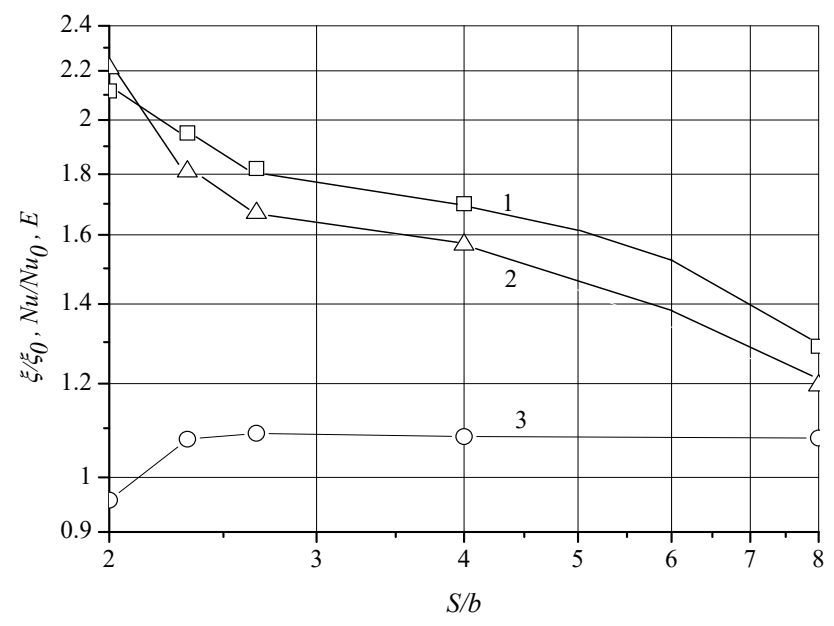

Figure 5. Relative heat transfer $N u / N u_{0}$ (1), friction factor $\xi / \xi_{0}(2)$ ratio, and thermal-hydraulic efficiency $E=\left(N u / N u_{0}\right) /\left(\xi / \xi_{0}\right)(3)$ versus the relative notching step $S / h$.

The numerical results presented by Isaev S. A. et al. [18] were obtained for the periodic duct section with the row of inclined to the main flow oval-trench dimples. The other parameters of the simulation were as follows: the length of the periodic domain $8 h_{c h}$, the width of the duct $9 h_{c h}$, where the $h_{c h}$ is the height of the domain. The oval-trench dimples had a length of 7.05b, width $b \approx h_{c h}$, depth $0.25 b$, and the edge rounding radius was $0.21 b$. The study on the attack angle influence $\left(\varphi=1-89^{\circ}\right)$ has shown that the area of the flow detachment decreases as the value of attack angle increases, while there was observed the growth of the reversal flow intensity in the dimple. The maximal friction factor augmentation was observed for the value of the attack angle of $\varphi=60^{\circ}$, while the thermal performance of such surface was in the direct proposition to the friction factor growth.

The extended results of [18] were presented in the work [19]. The value of the relative notching step $S / h$ was varied in the range $S / h_{c h}=2-8$. The extreme values of local transverse, longitudinal, and vertical velocities experienced a reduction while the attack angle was increased from the value $\varphi=45^{\circ}$ to $\varphi=65^{\circ}$ for the fixed relative length of the section $S / h_{c h}=8$. Thus, the intensity of the vortex generation decreases. The results agreed with the ones presented in [18]. However, while the value $S / h_{c h}=8$ was decreased to the value of $S / h_{c h}=2$, the attack angle value at which the extreme local velocities magnitudes were observed was found $\varphi=60^{\circ}$.

A more thorough investigation of the influence of the relative periodic section length on thermohydraulic properties of the airflow in the plain-parallel duct was presented in [20].

It was also shown that the notching step value decrease from $S=8 h_{c h}$ to $2 h_{c h}$ promotes the heat transfer augmentation from the magnitude of 1.18 up to 2.27 (Figure 5) relative to the smooth surface values, whereas the friction factor augmentation values rise from $\xi / \xi_{0}=1.18$ to 2.23 . Thus, the respective thermal-hydraulic efficiency coefficient $E=1.1$ could be achieved.

The results presented in [11-20] shall be verified experimentally.

Experimental studies on friction factor and heat transfer in ducts with the dimpled surfaces where the dimples had oval or oval-trench shape were carried out in a limited number of works by Burtsev, 
S.A., et al. [21], Voskoboynik, A.V., et al. [22], Voropaev, G.A., et al. [23], Sergievsky, E.D., et al. [24] and Popov, I.A., et al. [25]. However, in contrast to the numerical studies cited above, the dimples on the heat transfer surface were manufactured differently. The rounding of the dimple edge, as well as the dimple contours, considerably differed from the model used in the numerical studies.

The analysis of streamlines and values of local friction factors for the flow over the oval-trench dimple (Figure 6a,b) presented in [3-11] revealed the velocity stagnation areas in the vicinity of the front dimple edge. This has resulted in the local decrease of the heat transfer coefficient magnitudes. The elongation of the spherical dimple to the oval and oval-trench shape inevitably accompanies with the noted problem.

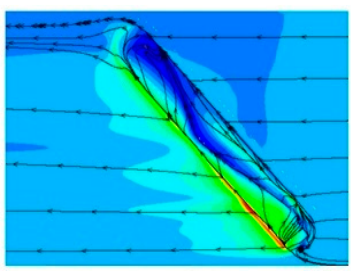

(a)

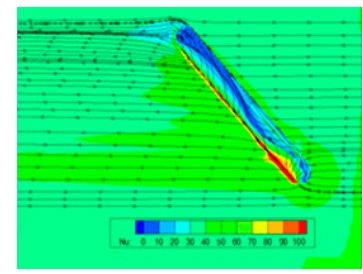

(b)

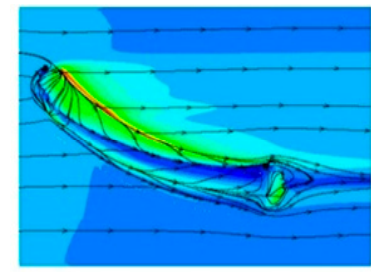

(c)

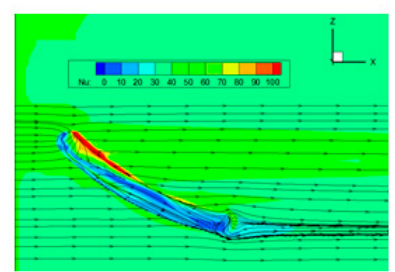

(d)

Figure 6. Streamlines for the flow over the oval-trench (a) and oval-arched (c) dimples and local heat transfer coefficients values $(\mathbf{b}, \mathbf{d})$, respectively.

The oval-arched dimple shape (Figure 7) has been proposed to settle the described problem [26]. The tangent line to the centerline for such dimple has to be 45 and 0 degrees at the begging and at the end of the dimple, respectively. Thus, the attack angle with respect to the main flow varies within the indicated range along the dimple. The other geometrical parameters of the dimple have to be settled as follows: $l_{d} / b=5.57-6.78 ; h / b=0.18-0.37 ; r=0.025 b$ (Figure $7,[26]$ ).
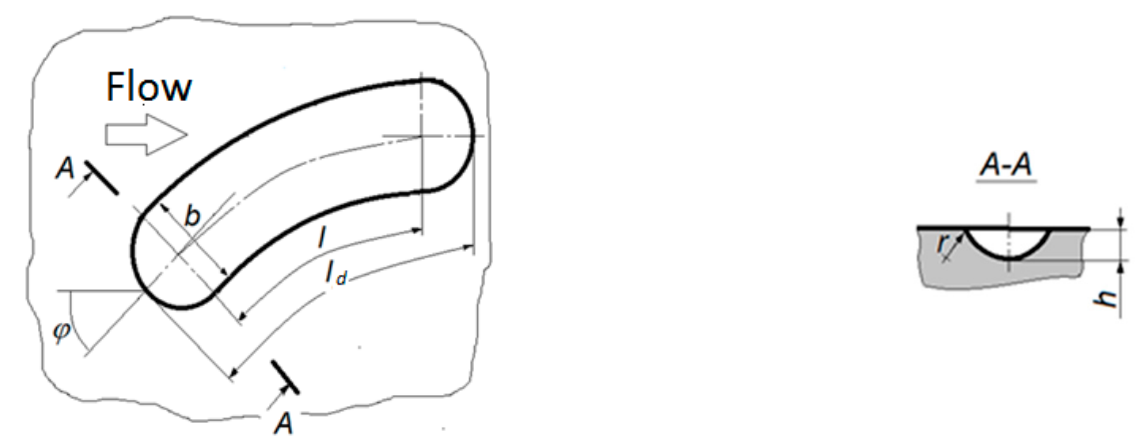

Figure 7. Characteristic scheme of the oval-arched shaped dimple: $l$-a length of the trench part, $\mathrm{mm}$; $l_{d}$ - a total length, $\mathrm{mm} ; h$-a depth, $\mathrm{mm} ; b$ - a width, $\mathrm{mm} ; r$ - edge rounding radius, $\mathrm{mm} ; \varphi$ - the angle of attack (to the main flow), deg, of the dimple and $S$ - a notching step; $A-A$-cross-section.

The results of the numerical simulation of the flow over the oval-arched dimple (Figure $6 c, d$ ) showed the absence of the flow stagnation zones in the dimple. Thereby, the rise of the local heat transfer coefficients and flow velocity magnitudes have been observed.

\section{Experimental Methodology}

The experimental investigation was carried out on the experimental air stand presented in Figure 8. The airflow was maintained by the compressors $1-3$ and receivers 10,12 . The required pressure in the air ducts has been attained by filling the receivers from one of the compressors before carrying out the experiments. The desired compressors were incorporated by the electromagnetic two-position valves $4,5,6$, and valves 9 . The air has been filtrated by the filtration module 7 and dehumidified by dehumidifier 8 . The air volume flow rate through the working area 19 was regulated by valve 11 , 
solenoid valve two-position pilot action 13, incorporated by the, operated by means of the control panel 21. Fine-tuning of the required flow and pressure on the working area 19 is regulated by the electrically operated ball valve for bypass 15 and the electrically operated ball valve 14, remotely controlled by controller 20. The volume flow rate was measured by the ultrasonic flow meter 16 . The temperature of the heat carrier was regulated by in-line electric heater 17 with energy source 18 . The working area has been heated up by an electrical resistance metal film substrate.

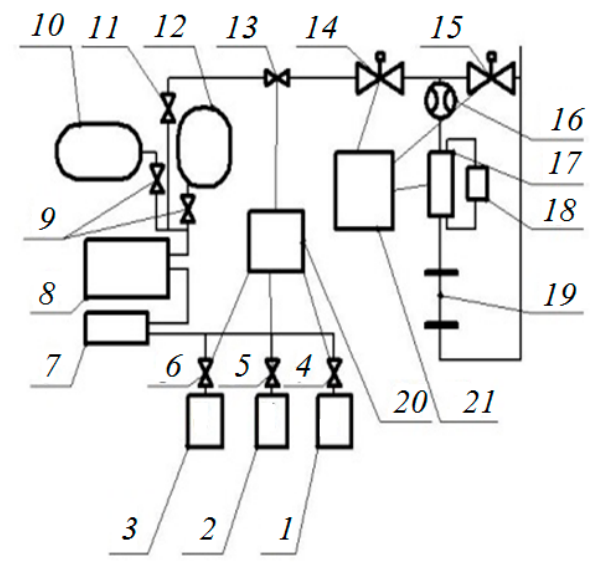

Figure 8. Scheme of the experimental stand: 1, 2, 3-compressors (capacity $2400 \mathrm{~L} / \mathrm{min}, 1400 \mathrm{~L} / \mathrm{min}$, $880 \mathrm{~L} / \mathrm{min}$ ); 4, 5, 6, 13-solenoid valve two-position pilot action; 7-filtration module for cleaning compressed air; 8-dehumidifier; 9, 11-valves; 10, 12-receivers; 14 -electrically operated ball valve; 15-electrically operated ball valve for bypass; 16 - ultrasonic flowmeter-gas meter; 17-heater; 18—energy source for heater; 19—working area; 20—controller; 21—valve control panel 4, 5, 6, 13.

The working area had the shape of a narrow rectangular duct with the replaceable dimpled surface. The flow stabilization ducts with the length of 50 equivalent diameters were added before and after the working area. The static pressure taps were installed in the lid of the working area in order to evaluate the friction factor value (1). The local heat transfer coefficients (2) were calculated by evaluating the values of the chromel-type thermocouples installed under the heat transfer surfaces.

$$
\begin{gathered}
\xi d=\frac{2 \cdot \Delta P \cdot d e}{\rho \cdot \bar{V} \cdot L}, \\
\alpha=\frac{\dot{Q}}{\Delta T \cdot A},
\end{gathered}
$$

where $\rho$-the air density, $\mathrm{kg} / \mathrm{m}^{3}$, mean airflow velocity, $\mathrm{m} / \mathrm{s} ; A$-area of the cross-section, $\mathrm{m}^{2}$, $P$-perimeter of the cross-section, $\mathrm{m} ; L$-the length of the working area, $\mathrm{m} ; d_{e}=4 \cdot \mathrm{A} / \mathrm{P}$ equivalent diameter of the duct, $\mathrm{m} ; \Delta T=T_{w}-T_{f}$-surface to flow mean temperature difference, $\mathrm{K} ; \mathrm{Q}$-heat flow supplied from the surface, Watt; $Q$ determined by the colorimetric method and controlled by the magnitude of the electric current supplied to the heating film, takes into account heat energy losses. A heat exchange surface was calculated without taking into account a surface extended by the dimples (relative to a smooth surface).

The desired parameters were determined according to the experimental investigation: Nusselt number $N u_{d}=\alpha d_{e} / \lambda$, Reynolds number $R e_{d}=\rho \cdot \bar{V} \cdot d_{e} / \mu$, Prandtl number $\operatorname{Pr}=\mu c / \rho$, where $\lambda$-heat conductivity, $\mathrm{W}(/ \mathrm{m} \cdot \mathrm{K}), \mu$-dynamic viscosity, Pa.s. The equivalent diameter of the duct $d_{e}$ was used as the characteristic dimension, and the average temperature of the heat carrier in the duct was used as the characteristic temperature. Using similarity numbers, experimental data processing is obtained and recommendations are given on the rational dimensions and ranges of applicability of the obtained data for heat transfer intensification and increase of heat-hydraulic efficiency. 
The experiments were carried out for the following dimensionless flow parameters $-\mathrm{Pr}=0.71$, $R e_{d}=3200-9 \cdot 10^{4}$ for the heat transfer coefficient determination and $R e=500-10^{4}$ for the friction factor measurements, and relative geometrical parameters of the heat transfer surfaces- $h / b=0.25-0.33$, $l_{d} / b=7$, and $\varphi=45^{\circ}$. Before carrying out the experiments for the flow in the ducts with the dipped surfaces, the comparison of the test experiments for the smooth duct was conducted. The friction factor and heat transfer coefficient measurements for the smooth duct were compared with the related values obtained by the widely used Blasius: $\left(\xi_{d 0}=0.3164 / R e_{d}^{0.25}\right)$ and Mikheev: $\left(N u_{d 0}=0.018 \cdot R e_{d}^{0.8}\left(\frac{T f}{T w}\right)^{0.5}\right)$ equations for the turbulent flow, respectively. The $T_{w}$ and $T_{f}$ is the wall and the mean flow temperatures. The maximal deviation of the measured and the calculated data was 10-15\%, which was found acceptable for further experiments.

\section{Experimental Results}

\subsection{Analysis of the Flow Structure}

The experimental visualization of the flow over the dimple was carried out by the ink supplied through the special ports mounted before the dimple.

The results of the flow visualization for the various Reynolds number values is presented in Figures 9 and 10.
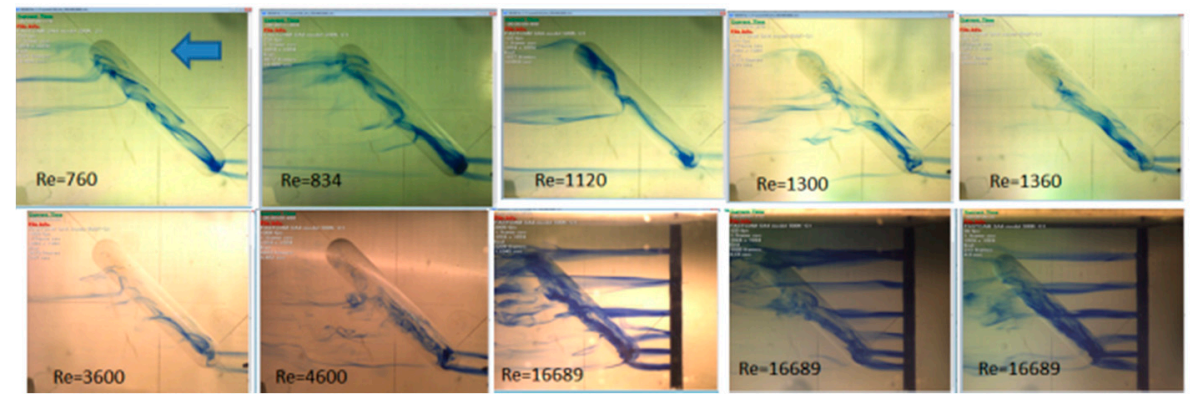

Figure 9. Visualization of flow over the oval-trench dimple for $h / b=0.5, l_{d} / b=7$ for the various Reynolds number values.
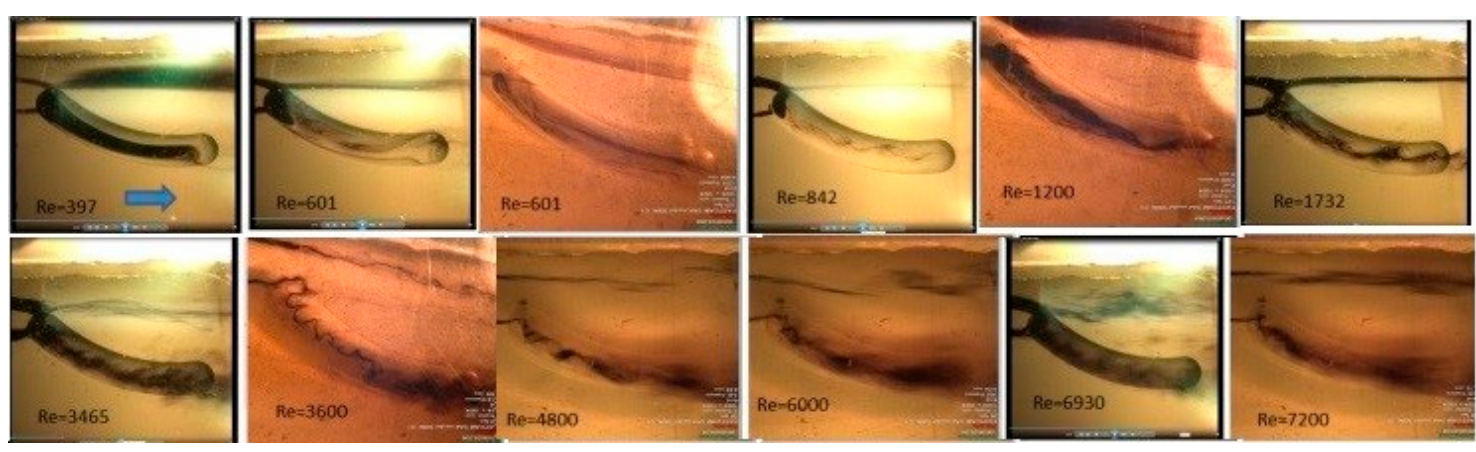

Figure 10. Visualization of flow over the oval-arched dimple for $h / b=0.5, l_{d} / b=7$ for the various Reynolds number values.

The visual comparison of the flow streamlines (Figure 6a) and results of the visualization (Figure 9) of the flow over the oval-trench dimple confirms the existence of the flow stagnation zone in the areas in the vicinity of the front dimple edge. These areas with the low-velocity magnitudes decrease the overall thermal performance of a dimpled surface. The flow deceleration in the dimple due to the dimple elongation could be overcome by the flow redirection through the dimple shape change. The visualization of the flow in the duct with the oval-arched dimple was made in order to assess the prospects of the oval-arched dimple for the heat transfer enhancement. 
The analysis of the presented results is served to confirm the acceleration of the velocity magnitudes in the dimple, which has been shown numerically. The flow stagnation areas were not observed.

The determination of the overall friction factor values for the flow in a duct with a single row and -multirow dimple arrangement on the bottom surface of the duct was carried out on the experimental stand Figure 8. The working area had a length of $L=0.188 \mathrm{~m}$, a width $B=0.098 \mathrm{~m}$, and the height $H=0.0017 \mathrm{~m}$ for the multirow dimple arrangement and $L=0.27 \mathrm{~m}, B=0.021 \mathrm{~m}, H=0.003 \mathrm{~m}$ for the single row dimple arrangement.

The comparison of the friction factor values for the flow over the surfaces with the oval-trench and oval-arched dimples $\left(h / b=0.25, l_{d} / b=7\right)$ with the multi-row dimple arrangement presented in Figure 11.

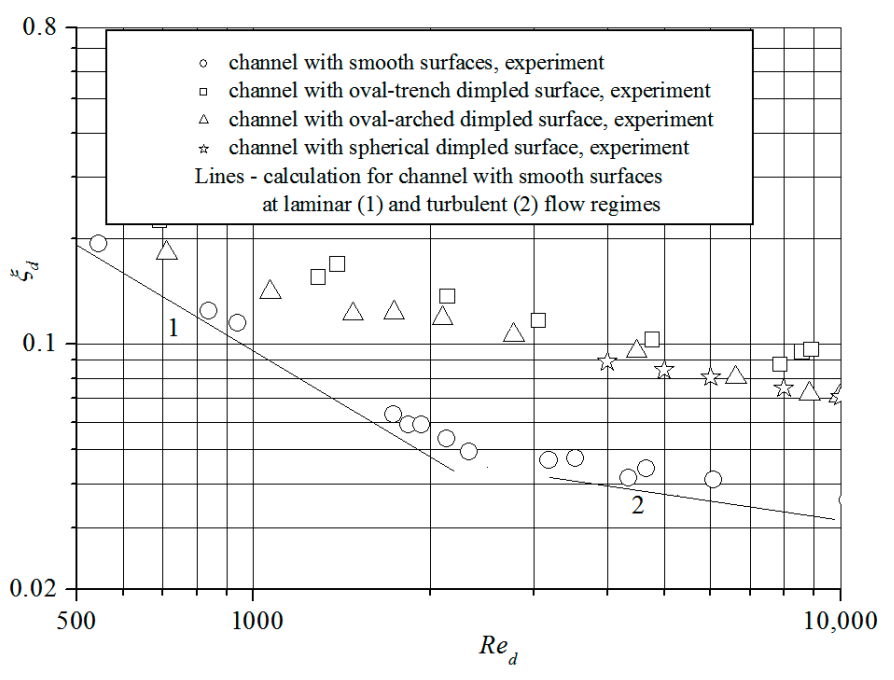

(a)

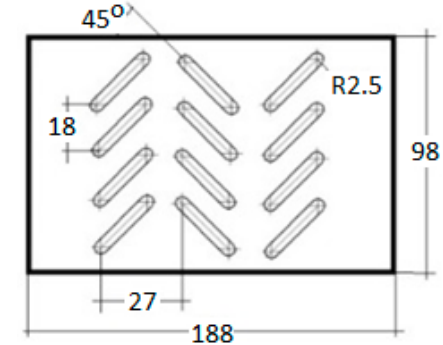

(b)

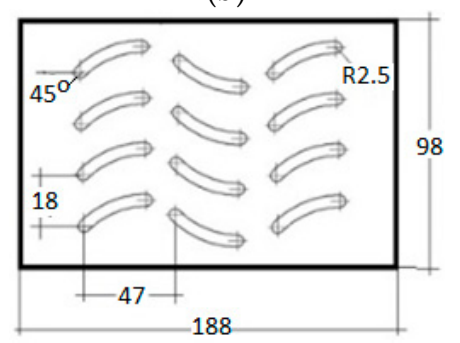

(c)

Figure 11. Friction factor coefficients versus Reynolds number values (a) for the surface with the multi-row arrangement of oval-trench (b) and oval-arched (c) dimpled surfaces. Sizes are in $\mathrm{mm}$.

Similar results were obtained for the single-row dimple arrangement and presented in Figure 12.

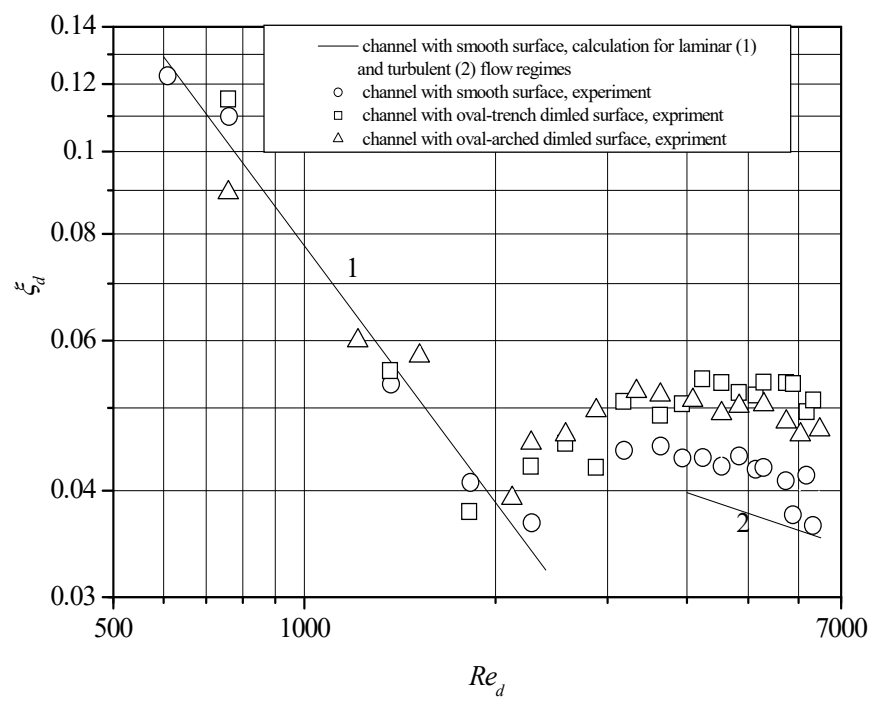

(a)

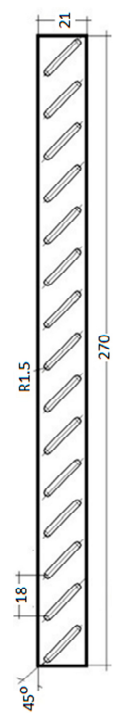

(b)

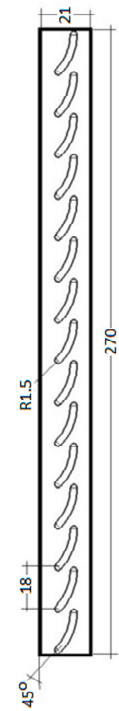

(c)

Figure 12. Friction factor coefficients versus Reynolds number values (a) for the surface with the single-row arrangement of oval-trench (b) and oval-arched (c) dimpled surfaces. Size are in $\mathrm{mm}$. 
The comparison results revealed that the friction factor values for the flow over the surfaces with the oval-arched dimples are lower, by 10-13\% than for the oval-trench dimpled surface, with the same geometrical parameters. Wherein, the friction factor values are at the same values as for the flow over the surface with the hemispherical dimples.

\subsection{Analysis of the Flow Structure}

Preliminary results of the infrared imaging comparison of the dimpled surfaces have revealed the better thermal performance of the oval-arched dimpled surfaces. The flow to surface temperature difference was found to be 5-20\% lower than for the surface.

Those findings are supported by the results of the experimental determination of the heat transfer coefficient values for the surfaces with the single- and multi-row arrangement of oval-arched and oval-trench dimples (Figure 13).

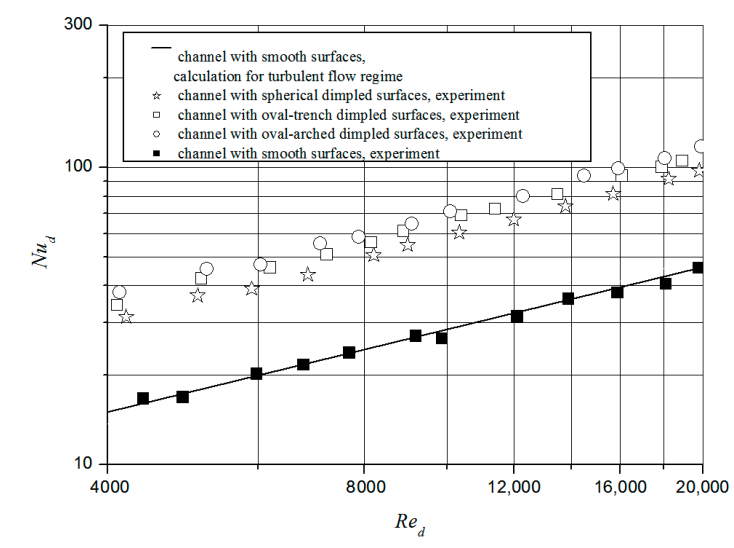

(a)

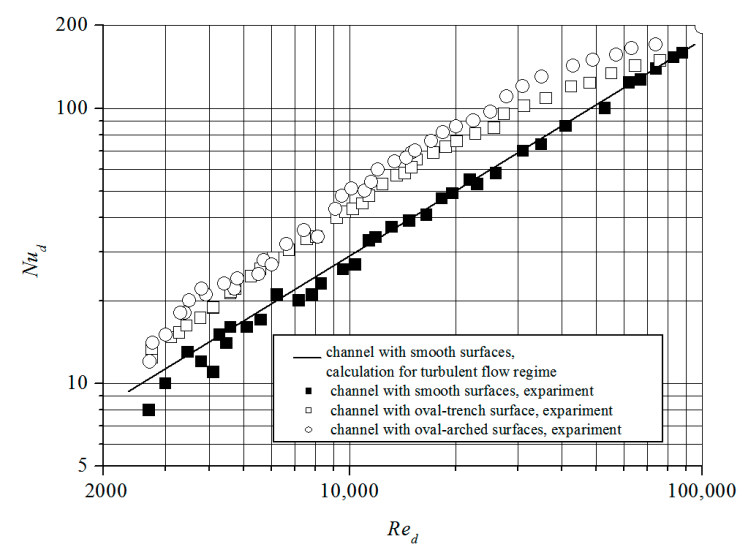

(b)

Figure 13. Heat transfer coefficient values for the surfaces with a multi-row (a) and single row (b) dimple arrangement of the oval-trench and oval-arched dimples.

Analysis of the results, presented in Figure 13 reveals the average heat transfer coefficients values for the surfaces with oval-trench dimples are 5-10\% lower than the ones for the surfaces with the oval-arched dimples $\left(h / b=0.33, l_{d} / b=7\right)$. Moreover, the thermal performance of the oval-arched dimple surfaces exceeds the heat transfer coefficient values specified for the surface with the hemispherical dimples for the same spot area of a single dimple.

The thermal performance of the dimpled surface was evaluated by comparing the values of the friction factor augmentation $\xi / \xi_{0}$, heat transfer augmentation $N u / N u_{0}$, and the thermal-hydraulic performance $E=\left(N u / N u_{0}\right) /\left(\xi / \xi_{0}\right)$. The subscript 0 is denoted for the smooth surface.

The results of the comprehensive comparison are listed in Table 1.

Table 1. Comparison of experimental data on the level of heat transfer and friction factor augmentation for the flow over the dimpled surfaces of various type.

\begin{tabular}{|c|c|c|c|c|c|c|c|c|}
\hline \multirow[t]{2}{*}{$\begin{array}{c}\text { Surface } \\
\text { Type }\end{array}$} & \multirow{2}{*}{$\begin{array}{l}\text { Dimple } \\
\text { Type }\end{array}$} & \multicolumn{2}{|c|}{$\begin{array}{c}\text { Relative } \\
\text { Dimple Size }\end{array}$} & \multicolumn{2}{|c|}{ Flow Parameters } & \multirow[t]{2}{*}{$N u / N u_{0}$} & \multirow[t]{2}{*}{$\xi / \xi_{0}$} & \multirow[t]{2}{*}{$E_{\max }$} \\
\hline & & $h / b$ & $l_{d} / b$ & $R e_{d}$ & $\operatorname{Pr}$ & & & \\
\hline \multirow{2}{*}{$\begin{array}{c}\text { Multi } \\
\text { row }\end{array}$} & Oval-trench & \multirow{2}{*}{0.25} & & \multirow{2}{*}{$4 \cdot 10^{3}-2 \times 10^{4}$} & \multirow{4}{*}{0.72} & $2.3-2.43$ & $2.5-3$ & 0.97 \\
\hline & Oval-arched & & 7 & & & $\sim 2.5$ & $2.3-2.6$ & 1.11 \\
\hline \multirow{2}{*}{$\begin{array}{l}\text { Single } \\
\text { rowe }\end{array}$} & Oval-trench & \multirow{2}{*}{0.33} & & \multirow{2}{*}{$3200-9 \times 10^{4}$} & & $1.2-1.55$ & $\sim 1.4$ & 1.11 \\
\hline & Oval-arched & & & & & $1.2-1.71$ & $\sim 1.25$ & 1.37 \\
\hline
\end{tabular}




\subsection{Verification and Recommendation}

The comparison of the outlined values was carried out with the results of the numerical simulation [20] for similar flow and geometrical parameters (recalculating the Reynolds number values $\operatorname{Re}=\rho \bar{V} d_{e} / \mu$ ). A good data value agreement was observed. The friction factor augmentation value in the numerical simulation was found to be $\xi / \xi_{0}=1.39$, while the experimental measurements have revealed the value of $\xi / \xi_{0}=1.4$. The corresponding values of the heat transfer augmentation for the numerical and experimental measurements were found to be $N u / N u_{0}=1.54$ and $N u / N u_{0}=1.55$, respectively. Thus, the data values difference is within $1 \%$.

The presented results on the heat transfer and friction factor augmentation for the flow over the surfaces with the oval-trench and oval-arched dimple arrangements could be used for the design and manufacturing of various cooling and thermal apparatus. The increased appliance of the oval-trench and oval-arched dimpled surfaces was found in the systems of heat exchangers of systems for heat recovery or utilization or on plates of the plate and shell-and-plate heat exchangers with oval (flattened) tubes. The discussed dimples could be on the heat transfer surfaces by means of cold stamping, casting, 3D prototyping, mechanical cutting, etc.

Due to the presence of extremes in the dependencies $\xi_{d}$ and $N u_{d}$ on the geometric parameters of vortex generators $\left(h / b, S / b, l_{d} / b, \varphi\right)$, a large set of criterion equations or complex equations is required to predict their values. For a more technically interesting range of geometric parameters (providing the large thermal performance), $h / b=0.0625-0.25, S / b=2-8, l_{d} / b=3-7, \varphi=45-60$, and turbulent flow regime, it is proposed to use criteria equations to estimate the coefficients of hydraulic resistance and heat transfer in channels with single row arrangement of oval-trench dimples:

$$
\begin{gathered}
\xi_{d}=1.23(h / b)^{0.22}(S / b)^{-0.385}\left(l_{d} / b\right)^{-0.023}(\varphi / 90)^{-0.027} / \operatorname{Re}_{d}{ }^{0.25} \\
N u_{d}=0.162 \cdot \operatorname{Re}_{d}^{0.72} \operatorname{Pr}^{0.4}(h / b)^{0.317}(S / b)^{-0.336}\left(l_{d} / b\right)^{0.083}(\varphi / 90)^{-0.075}
\end{gathered}
$$

Equations (3) and (4) were elaborated on the cumulative generalization of the experimental and numerical data presented in the current paper.

The reference temperature-the air temperature at the entrance to the duct, the reference velocity - the average mass flow velocity in the duct, characteristic linear dimention-the hydraulic diameter of the channel. The equations allow for predicting thermal and hydraulic characteristics with a deviation of up to $20 \%$.

\subsection{Artificial Neural Network Application}

The literature analysis reveals the potential for the data prediction on the basis of the experimental and numerical results database $[27,28]$. An analysis of the applicability of artificial neural networks for estimating the coefficients of hydraulic resistance and heat transfer for flow over excavated surfaces is carried out.

The database contained 200 data points, presented in [11-16,18-20]. A model of the artificial neural works contained two hidden layers of six and four neurons. The program code was written in Python 3.0 with the use of Keras and Tensor flow Libraries. The data were separated on test and training set at the ratio of 20 to 80 percent in order to overcome the over-prediction problem. By means of the backpropagation, the weight factors of the model were determined.

The comparison of the friction factor values predicted by the model of the artificial neural network with the results of the numerical simulation was found to be in a good agreement: $\xi / \xi_{0}=1.39$ for the numerical simulation and $\xi / \xi_{0}=1.46$ for the neural network model; $N u / N u_{0}=1.54$ for the numerical simulation and $N u / N u_{0}=1.66$ for the model of a neural network $\left(h / b=0.33, S / b=6, l_{d} / b=7, \varphi=45^{\circ}\right.$, $\left.R e=10^{4}\right)$. The mean square error over the whole database was within 5-8\% for the hydraulic resistance and heat transfer augmentation values both for the test and training datasets. However, it shall be noticed that a more thorough and comprehensive analysis is required. 
It is recommended to use the model of the artificial neural network when predicting thermohydraulic parameters by means of computer program for the use of heat exchange equipment design and ensuring increased accuracy of predicting their parameters.

\section{Conclusions}

A literature review outlining existing findings on the friction factor and heat transfer augmentation in the narrow ducts with single-row arrangements of the oval-trench dimples on the heat transfer surfaces is presented. The rational relative geometrical parameters of the dimple which provides higher thermal performance of the heat transfer surface for the turbulent flow were revealed: relative elongation of dimple $l / b=5.57-6.78$ and relative depth $h / b=0.18-0.37$, while the value of the attack angle to the mean flow is fixed $\varphi=(45-60)^{\circ}$. The outlined geometrical parameters of the dimple were patented.

The experimental investigation of the flow over the single and multi-row oval-trench and oval-arched dimpled surfaces was carried out $\left(\mathrm{Pr}=0.71, \operatorname{Re}_{d}=3200-9 \times 10^{4}\right.$ for the heat transfer coefficient measurements and $R e_{d}=500-10^{4}$ for the friction factor determination, $h / b=0.25$ and $\left.0.33, l_{d} / b=7, \varphi=45^{\circ}\right)$. The close agreement between the numerical and the experimental results was indicated.

The maximum heat transfer augmentation for the single row arrangement of the oval-trench and oval-arched dimples was found to be $\mathrm{Nu} / \mathrm{N} u_{0}=1.2-1.7$, whereas, for the multirow dimple arrangement, the corresponding values were $N u / N u_{0}=2.3-2.5$. The application of the oval-arched dimples yields a 5-10\% larger thermal performance than the oval-trench one under the same conditions.

It was shown that the friction factor augmentation for such surfaces does not exceed the corresponding heat transfer augmentation $\left(\xi / \xi_{0}=1.25-1.4\right.$ for the single row dimple arrangement, and $\xi / \xi_{0}=2.3-3$ for the multirow dimple arrangement). The oval-arched dimpled surfaces yield 10 to $15 \%$ lower hydraulic losses than the surfaces with the oval-trench dimple arrangements. Thus, the thermal performance of the heat transfer surfaces could be increased up to $23 \%$ and $14 \%$ for the single- and multirow dimple arrangements, respectively.

The generalized equations for the turbulent flow in the duct with the dimpled surfaces are presented. The equations allow for predicting the values of friction factor and heat transfer coefficients in ducts with single-row oval-trench dimple arrangements with a maximal deviation of up to $20 \%$ (for $h / b=0.0625-0.25, S / b=2-8, l_{d} / b=3-7, \varphi=45-60$ ).

The artificial neural network application allows for simplifying the procedure for determining the values of $\xi / \xi_{0}$ and $N u / N u_{0}$ over the entire range of changes in the defining mode and geometric parameters by means of the related program code. The mean square error over the whole database was within $5-8 \%$ for the hydraulic resistance and heat transfer augmentation values both for the test and training datasets.

The obtained results are recommended for use in predicting heat transfer and hydraulic resistance in the channels of heat exchange equipment when using oval-arch and oval-trench excavations.

Author Contributions: Conceptualization, S.I.; Formal analysis, S.I. and I.P.; Investigation, A.M. and A.S.; Methodology, A.S. and I.P.; Project administration, S.I.; Software, S.I. and A.S.; Supervision, S.I. and I.P.; Visualization, A.S.; Writing-original draft, I.P.; Writing—review \& editing, I.P. All authors have read and agreed to the published version of the manuscript.

Funding: Work was supported by the Ministry of Science and Higher Education of the Russian Federation under agreement 075/03/2020/051/3 (experiments), Russian Scientific Foundation grant number 19-19-00259 (numerical simulation) and Russian Foundation for Basic Research grant number 19-38-90077 (artificial neural network).

Conflicts of Interest: The authors declare no conflict of interest. 


\section{Nomenclature}

$\begin{array}{ll}A & \text { area of the cross-section of the duct, } \mathrm{m}^{2} ; A=B \cdot h_{c h} \\ B & \text { a duct width, } \mathrm{m} ; \\ b & \text { a dimple width, } \mathrm{m} ; \\ c & \text { heat capacity, } \mathrm{J} / \mathrm{kg} \cdot \mathrm{K} \\ d_{e} & \text { equivalent diameter of the duct, } \mathrm{m} ; d_{e}=4 \cdot A / P \\ h & \text { a dimple depth, } \mathrm{m} ; \\ h_{c h} & \text { a duct height, } \mathrm{m} ; \\ L & \text { a duct length, } \mathrm{m} ; \\ l & \text { a length of the trench part of the dimple, } \mathrm{m} ; \\ l_{d} & \text { a total length, } \mathrm{m} ; \\ m & \text { mass flow rate, } \mathrm{kg} / \mathrm{s} \\ P & \text { perimeter of the cross-section, } \mathrm{m} \\ Q & \text { heat flow supplied from the surface, } \mathrm{W} \\ R & \text { the dimple forming radius, } \mathrm{m} \\ r & \text { an edge rounding radius, } \mathrm{m} ; \\ S & \text { a notching step, } \mathrm{m} \\ T & \text { temperature, } \mathrm{K} \\ T_{f} & \text { a temperature of the flow, } \mathrm{K} \\ T_{w} & \text { a temperature of the wall, } \mathrm{K} \\ \bar{V} & \text { mean flow velocity, } \mathrm{m} / \mathrm{s} ; \bar{V}=m /(\rho \cdot A) \\ G \text { reek symbol: }\end{array}$

$\alpha \quad$ a heat transfer coefficient, $\mathrm{W} / \mathrm{m}^{2} \mathrm{~K} ; \alpha=Q / \Delta T \cdot A$

$\alpha_{0} \quad$ a heat transfer coefficient for the smooth surface, $\mathrm{W} / \mathrm{m}^{2} \mathrm{~K}: \alpha_{0}=Q / \Delta T \cdot A$

$\Delta P \quad$ a pressure drop, $\mathrm{Pa}$

$\Delta T \quad$ surface to flow mean temperature difference, $\mathrm{K} ; \Delta T=T_{w}-T_{f}$

$\lambda \quad$ a heat conductivity coefficient, $\mathrm{W} / \mathrm{m}^{2} \mathrm{~K}$

$\mu \quad$ a dynamic viscosity, Pa.s

$\xi \quad$ a Darcy-Weibach friction factor; $\xi=2 \cdot \Delta \mathrm{Ph}_{\mathrm{ch}} /(\rho \bar{V} \cdot L)$

$\xi_{0} \quad$ a friction factor value for the smooth surface; $\xi_{0}=2 \cdot \Delta P \cdot h_{c h} /(\rho \overline{V \times} L)$

$\xi_{d} \quad$ a friction factor value for the dimpled surface; $\xi_{d}=2 \cdot \Delta P \cdot d_{e} /(\rho \overline{V \times} L)$

$\xi_{d 0} \quad$ a friction factor for the surface without the dimples; $\xi_{d 0}=2 \cdot \Delta P d_{e} /(\rho \overline{V \times L})$

$\rho \quad$ a density of fluid, $\mathrm{kg} / \mathrm{m}^{3}$

$\varphi \quad$ an angle of attack of the dimple (to the main flow), deg,

Dimensionless complexes:

E a thermohydraulic efficiency coefficient; $E=\left(N u / N u_{0}\right) /\left(\xi / \xi_{0}\right)=\left(N u_{d} / N u_{d 0}\right) /\left(\xi_{d} / \xi_{d 0}\right)$

$E_{\max } \quad$ a maximal value of thermohydraulic efficiency

$\mathrm{Nu} \quad$ a Nusselt number, $N u=\alpha \mathrm{h}_{\mathrm{ch}} / \lambda$

$N u_{0} \quad$ a Nusselt number for the smooth surface, $N u_{0}=\alpha_{0} \mathrm{~h}_{\mathrm{ch}} / \lambda$

$N u_{d} \quad$ a Nusselt number for the dimpled surface, $N u_{d}=\alpha \mathrm{d}_{\mathrm{e}} / \lambda$

$N u_{d 0} \quad$ a Nusselt number for the surface without the dimples, $N u_{d 0}=\alpha_{0} d_{\mathrm{e}} / \lambda$

$N u / N u_{0} \quad$ a Nusselt number augmentation ratio; $N u / N u_{0}=N u_{d} / N u_{d 0}=\alpha / \alpha_{0}$

$\operatorname{Pr} \quad$ a Prandtl number, $P r=\mu c / \rho$

$\operatorname{Re} \quad$ a Reynolds number based on the duct height; $R e=\rho \bar{V} \mathrm{~h}_{\mathrm{ch}} / \mu$

$R e_{d} \quad$ a Reynolds number based on the equivalent diameter of the duct; $R e_{d}=\rho \bar{V} d_{\mathrm{e}} / \mu$

$\xi / \xi_{0} \quad$ a friction factor augmentation value; $\xi / \xi_{0}=\xi_{d} / \xi_{d 0}$

\section{References}

1. Rashidi, S.; Hormozi, F.; Sunden, B.; Mahia, O. Energy Saving in Thermal Energy Systems Using Dimpled Surface Technology-A review on mechanisms and applications. Appl. Energy 2019, 250, 1491-1547. [CrossRef]

2. Shchukin, A.V.; Kozlov, A.P.; Agachev, R.S.; Chudnovsky, Y.P. Intensification of Heat Exchange by Spherical Dimples under the Influence of Perturbing Factors; Kazan State Technical University: Kazan, Russia, 2003; p. 143. 
3. Khalatov, A.A. Heat Transfer and Hydrodynamics near Surface Depressions (Dimples); Instutute of Technical Thermal Physics: Kiev, Ukraine, 2005; p. 76.

4. Kiknadze, G.I.; Gachechiladze, I.A.; Alekseev, V.V. Self-Organization of Tornado-Like Jets in Flows of Viscous Continuous Media and Intensification of Heat and Mass Transfer; Publishing House of Moscow Power Engineering Institute: Moscow, Russia, 2005; p. 82.

5. Bystrov, Y.A.; Isaev, S.A.; Kudryavtsev, N.A.; Leontiev, A.I. Numerical Simulation of Vortex Heat Transfer Intensification in Tube Packages; Sudostroenie: Saint Petersburg, Russia, 2005; p. 398.

6. Dzyubenko, B.V.; Kuzma-Kichta, Y.A.; Leontiev, A.I.; Fedik, I.I.; Holpanov, L.P. Intensification of Heat and Mass Transfer on Macro-, Micro- and Nanoscales; CNIIATOMINFORM: Moscow, Russia, 2008; p. 532.

7. Gortyshov, Y.F.; Popov, I.A.; Olympiev, V.V.; Shchelchkov, A.V.; Kaskov, S.I. Thermohydraulic Efficiency of Perspective Methods of Heat Transfer Intensification in Heat Exchange Equipment Channels; Center of Innovative Technologies: Kazan, Russia, 2009; p. 531.

8. Sokolov, N.P.; Polishchuk, V.G.; Andreev, K.L. Heat Transfer and Hydraulics in Channels with Dimpled Surfaces; Polytechnic University Publishing House: Saint Petersburg, Russia, 2012; p. 288.

9. Gotovsky, M.A.; Demenok, S.L.; Medvedev, V.V.; Sivukha, S.M. Heat Transfer and Resistance of Channels with Dimpled Surfaces; Strata: Saint Petersburg, Russia, 2016; p. 211.

10. Leontiev, A.I.; Alekseenko, S.V.; Volchkov, E.P.; Dzubenko, B.V.; Dragunov, Y.G.; Isaev, S.A.; Koroteev, A.A.; Kuzma-Kichta, Y.A.; Popov, I.A.; Terekhov, V.I. Vortical Technologies for Power Engineering; Publishing House of Moscow Power Engineering Institute: Moscow, Russia, 2017; p. 350.

11. Isaev, S.A.; Leontiev, A.I.; Mityakov, A.V.; Pyshny, I.A. Intensification of Tornado Turbulent Heat Transfer in Asymmetric Wells on a Flat Wall. J. Eng. Phys. Thermophys. 2003, 76, 31-34.

12. Isaev, S.A.; Popov, I.A.; Leontiev, A.I.; Gultsova, M.E. Transformation and Intensification of Tornado-Like Flow in a Narrow Channel during Elongation of an Oval Dimple with Constant Area. Tech. Phys. Lett. 2015, 41, 606-612. [CrossRef]

13. Isaev, S.A.; Schelchkov, A.V.; Leontiev, A.I.; Gortyshov, Y.F.; Baranov, P.A.; Popov, I.A. Tornado-Like Heat Transfer Enhancement in the Narrow Plane-Parallel Channel with the Oval-Trench Dimple of Fixed Depth and Spot Area. Int. J. Heat Mass Transf. 2017, 109, 40-62. [CrossRef]

14. Isaev, S.; Leontiev, A.; Chudnovsky, Y.; Popov, I. Vortex Heat Transfer Enhancement in Narrow Channels with a Single Oval-Trench Dimple Oriented at Different Angles to the Flow. J. Enhanc. Heat Transf. 2018, 25, 579-604. [CrossRef]

15. Isaev, S.A.; Baranov, P.A.; Leontiev, A.I.; Popov, I.A. Intensification of a Laminar Flow in a Narrow Microchannel with Single-Row Inclined Oval-Trench Dimples. Tech. Phys. Lett. 2018, 44, 398-400. [CrossRef]

16. Isaev, S.A.; Leontiev, A.I.; Milman, O.O.; Popov, I.A.; Sudakov, A.G. Influence of the Depth of Single-Row Oval-Trench Dimples Inclined to Laminar Air Flow on Heat Transfer Enhancement in a Narrow Micro-Channel. Int. J. Heat Mass Transf. 2019, 134, 338-358. [CrossRef]

17. Isaev, S.A.; Leontiev, A.I.; Baranov, P.A.; Popov, I.A.; Shchelchkov, A.V.; Gortyshov, Y.F.; Skrypnik, A.N.; Mironov, A.A. Heat Exchange Surface. Patent No. 2684 303. Russian Federation, IPC7 F28F 3/04. Published: 05.04.2019. Byul. No. 10. Priority 13.06.2018. Available online: https://www.fips.ru/iiss/document.xhtml? faces-redirect=true\&id=a7dfd30ed95db609149ac796e1758915 (accessed on 27 September 2020).

18. Isaev, S.A.; Gritckevich, M.S.; Leontiev, A.I.; Popov, I.A.; Sudakov, A.G. Abnormals Intensification of Turbulent Separated Flow in Inclined Single-Row Oval-Trench Wells on the Wall of a Narrow Channel. High Temp. 2019, 57, 797-800. [CrossRef]

19. Isaev, S.A.; Gritskevich, M.S.; Leontiev, A.I.; Milman, O.O.; Nikushchenko, D.V. Acceleration of the Turbulent Flow in a Narrow Oblong Channel and Intensification of the Detached Flow when Compacting Single-Row Inclined Oval-Trench Wells on the Wall. Thermophys. Aeromech. 2019, 26, 697-702. [CrossRef]

20. Isaev, S.A.; Gritckevich, M.S.; Leontiev, A.I.; Milman, O.O.; Nikushchenko, D.V. NT Vortex Enhancement of Heat Transfer and Flow in the Narrow Channel with a Dense Packing of Inclined One-Row Oval-Trench Dimples. Int. J. Heat Mass Transf. 2019, 145, 118737. [CrossRef]

21. Kiselev, N.A.; Burtsev, S.A.; Strongin, M.M.; Vinogradov, Y.A. Experimental Study of Heat Transfer and Resistance of Complex-Shaped Dimples. In Fundamental and Applied Problems of Heat and Mass Transfer. Proceedings of the Anniversary Conference of the National Committee of the Russian Academy of Sciences on Heat and Mass Transfer; Publishing House of Moscow Power Engineering Institute: Moscow, Russia, 2017; pp. 124-127. 
22. Voskoboynik, A.V. Passive Control of the Formation of Vortex Structures inside a Semi-Cylindrical Dimples. Bull. Donetsk Natl. Univ. Ser. A Nat. Sci. 2009, 1, 173-182.

23. Voropaev, G.A.; Voskoboynik, A.V.; Voskoboynik, V.A.; Isaev, S.A. Visualization of Laminar Flow around Oval Dimples. Appl. Hydraul. Mech. 2009, 11,31-36.

24. Sergievsky, E.D.; Arbatsky, A.A. Intensification of Heat Exchange by Applying Oval Dimples to the Heat Exchange Surface. In Proceedings of the Fifth Russian National Conference on Heat Exchange, Moscow, Russia, 26-29 October 2010; Publishing House of Moscow Power Engineering Institute: Moscow, Russia, 2010; Volume 6, pp. 141-144.

25. Popov, I.A.; Shchelchkov, A.V.; Ryzhkov, D.V.; Ulyanova, R.A. Vortex Formation in Separated Flows on Surfaces with Dimples of Various Shapes. Proc. Akad. 2010, 3, 7-14.

26. Isaev, S.A.; Leontiev, A.I.; Baranov, P.A.; Popov, I.A.; Shchelchkov, A.V.; Gortyshov, Y.F.; Skrypnik, A.N.; Mironov, A.A. Heat Exchange Surface. Patent No. 2716 958. Russian Federation, IPC7 F28F 3/04. Published: 17.03.2020. Byul. No. 8. Priority 26.07.2019. Available online: https://www.fips.ru/iiss/document.xhtml? faces-redirect=true\&id=17825c9832e585d2fc610d2946294113 (accessed on 27 September 2020).

27. Jambunathan, K.; Hartle, S.L.; Ashforth-Frost, S.; Fontama, V.N. Evaluating Convective Heat Transfer Coefficients Using Neural Networks. Int. J. Heat Mass Transf. 1996, 39, 2329-2332. [CrossRef]

28. Zdaniuk, G.J.; Chamra, L.M.; Walters, D.K. Correlating Heat Transfer and Friction in Helically-Finned Tubes Using Artificial Neural Networks. Int. J. Heat Mass Transf. 2007, 50, 4713-4723. [CrossRef]

(C) 2020 by the authors. Licensee MDPI, Basel, Switzerland. This article is an open access article distributed under the terms and conditions of the Creative Commons Attribution (CC BY) license (http://creativecommons.org/licenses/by/4.0/). 